\section{Risk management in clinical practice. Part 9. Dental implants}

\author{
R. M. Palmer ${ }^{1}$
}

VERIFIABLE CPD PAPER

IN BRIEF
Risk factors associated with implant
failure and complications need to be
assessed for each patient individually.
- A thorough evaluation of adjacent teeth
and the mouth in general is required for
proper treatment planning.
Damage to adjacent structures is
avoidable but increasingly the subject of
clinical negligence claims.
Practitioners should be adequately
trained and follow the GDC guidelines.

Patients have high expectations of dental implants in terms of appearance, function and longevity. It is essential that these expectations are realistically managed and that treatment of the highest standard is provided. This involves very careful evaluation, including clinical and radiographic, and presentation of the pros and cons of treatment alternatives. Provision of a successful implant restoration requires many skills including a surgical procedure to place the implant in the best possible position and prosthodontic techniques to provide an aesthetic restoration in occlusal harmony with the rest of the dentition. Recognition of risk factors and long-term maintenance requirements are equally important. Clinicians involved in these treatments must obtain adequate training and develop skills through treatment of straightforward cases using well established protocols before embarking on more demanding cases.

\section{INTRODUCTION}

Dental implant treatment had a chequered history before the development of modern osseointegrated root form implants. Earlier designs such as blade implants made from

\begin{tabular}{l} 
RISK MANAGEMENT \\
IN CLINICAL PRACTICE \\
\hline 1. Introduction \\
2. Getting to 'yes' - the matter of consent \\
3. Crowns and bridges \\
4. Endodontics \\
5. Ethical considerations for dental \\
enhancement procedures \\
6a. Identifying and avoiding medico-legal \\
risks in complete denture prosthetics \\
6b. Identifying and avoiding medico-legal \\
risks in removable dentures \\
7. Dento-legal aspects of orthodontic \\
practice \\
8. Temporomandibular disorders \\
9. Dental implants \\
10. Periodontology \\
11. Oral surgery
\end{tabular}

'Professor of Implant Dentistry and Periodontology, King's College London Dental Institute, Department of Restorative Dentistry, Floor 25 Tower Wing, Guy's Hospital Campus, London, SE1 9RT

Correspondence to: Professor Richard Palmer Email: richard.m.palmer@kcl.ac.uk

\section{Refereed Pape}

Accepted 20 October 2009

DOI: $10.1038 /$ sj.bdj.2010.1033

${ }^{\circ}$ British Dental Journal 2010; 209: 499-506 materials that did not osseointegrate were often associated with inflammation, bone loss and catastrophic failure (Fig. 1). However, few clinicians were involved in providing this treatment and the number of legal cases was relatively small. This situation has changed dramatically. Dental implants now enjoy very high success rates and few complications, especially when provided by adequately trained individuals carrying out good levels of diagnosis, treatment planning and execution of the surgical and prosthodontic aspects of treatment (Fig. 2). Failure in any of these fundamental areas can lead to problems and potential litigation.

\section{EXAMINATION AND DIAGNOSIS}

It is increasingly common to see patients who seek dental implant treatment in the belief that it will solve all their problems, whether they are functional, aesthetic or simply because of bad experiences with their natural teeth. Patients have high expectations of dental implants possibly because of the large amount of information in the media, from the implant companies and the high cost of treatment. It is essential that their expectations are understood and that the clinician is confident that they can be met. Although implants may be immune to dental caries they are susceptible to the ravages caused by inflammation,

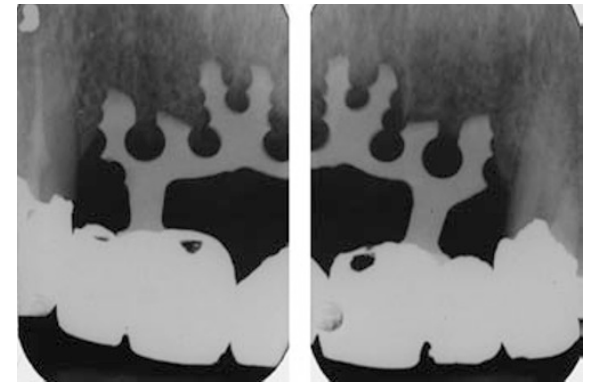

Fig. 1 A failed blade implant causing extensive bone loss

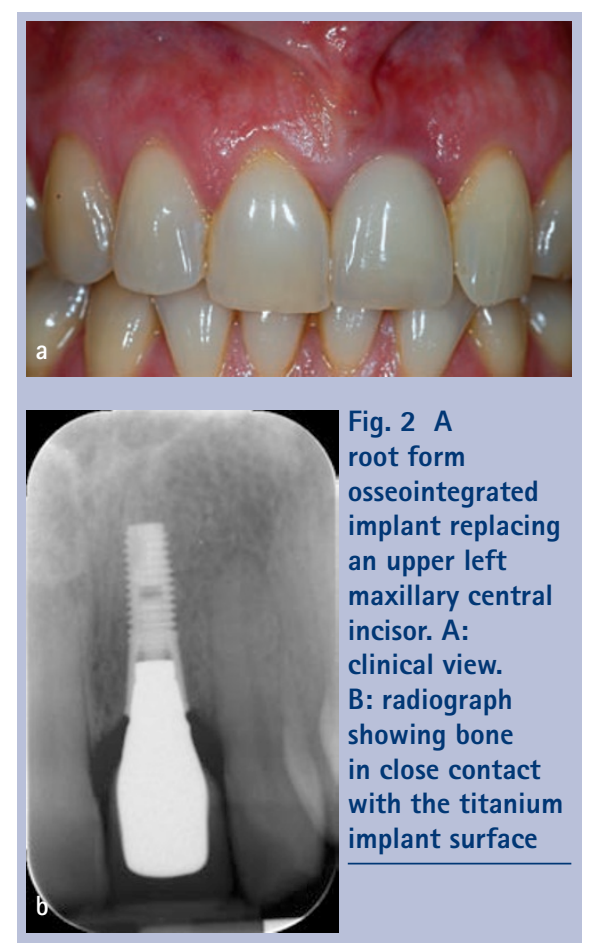


Table 1 Main risk factors associated with increased implant failure and complications

Irradiation

Tobacco smoking (especially high dose/duration)

Periodontitis

Endodontic lesions (eg apical granuloma)

Parafunction (eg bruxism)

Poor bone quality

smoking and parafunction (Table 1). The smoking bruxist with advanced periodontitis is not a good bet for dental implant treatment! There have also been reports of bone necrosis of the jaws in patients treated with bisphosphonates, but this is more of a problem in those who have received intravenous bisphosphonates rather than those on oral medication. One of the most important contraindications for implant treatment is the young child who has not completed growth. The ankylosed implant can become increasingly in infraocclusion following growth and eruption of the adjacent teeth.

It is absolutely essential that patients undergo a comprehensive history and examination to determine their main complaints and to avoid missing important diagnoses that will have a bearing on their dental management. Even common conditions such as caries, non-vital teeth and periodontitis can be missed when the dentist is focusing on the evaluation and provision of the more exciting aspects of dental implants. Implants placed in subjects with untreated periodontitis or close to periapical lesions are associated with higher failure rates. In this respect it is important to determine the reason for tooth loss and to manage all dental conditions as part of an overall treatment plan.

Aesthetic considerations can be of the greatest importance in some patients. Dental implant treatment will not necessarily provide the best aesthetics compared with the alternatives and patients are occasionally dissatisfied with the result. The coverage of the anterior teeth (and gingivae) by the lips during normal function and smiling should be carefully assessed. Any type of anterior maxillary prosthesis will be difficult to disguise in a patient with a high smile line (Fig. 3). An anterior restoration should also provide adequate lip support. The appearance of the planned restoration can be judged by providing a diagnostic set up or preferably a provisional prosthesis that can be worn by the patient and evaluated in their own time (Fig. 4). They may also serve extremely well as a model for a surgical stent or guide to assist in the optimal placement of the implants, and as a transitional restoration during the treatment programme.

\section{Evaluation of the edentulous ridge}

The height, width and contour of the edentulous ridge can be visually assessed and carefully palpated for the presence of concavities/depressions. However, accurate assessment of the underlying bone width is difficult, especially where the overlying tissue is fibrous. Clinical techniques such as ridge mapping have been advocated but this is prone to error and the advent of high quality tomography has made it almost obsolete. The clinician needs to gather sufficient information to determine whether there is sufficient bone for implant placement or whether the patient needs to be advised that bone augmentation is required.

The distance between the edentulous ridge and the opposing dentition should be measured to ensure that there is adequate room for the restorative components. The angulation of the ridge and its relationship to the opposing dentition is also important. Proclined ridge forms will tend to lead to proclined placement of the implants, which could affect aesthetics and loading. Large horizontal discrepancies between the jaws, for example the pseudo class III jaw relationship following extensive maxillary resorption, may not be suitable for treatment with fixed bridges.

The clinical examination of the ridge also allows assessment of the soft tissue thickness, which is important for the attainment of good aesthetics (Fig. 5). Keratinised tissue which is attached to the edentulous ridge will also generally provide a better peri-implant soft tissue than non-keratinised mobile mucosa. The length of the edentulous ridge can be measured to give an indication of the possible number of implants that could be accommodated. However, this also requires reference to radiographs to allow a correlation with available bone volume and the diagnostic set-up for the proposed tooth location. The aim is to provide an

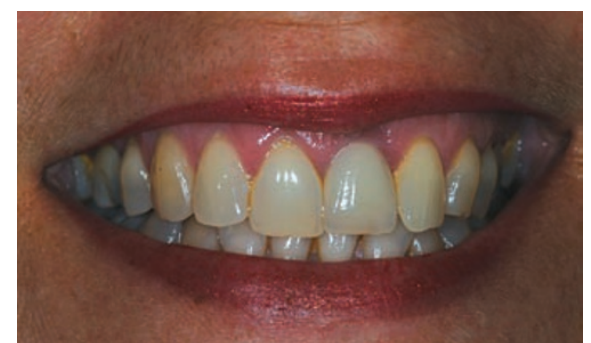

Fig. 3 The same patient as in Figure 2 showing a high smile line revealing a lot of gingival tissue and placing high demands on the aesthetic outcome

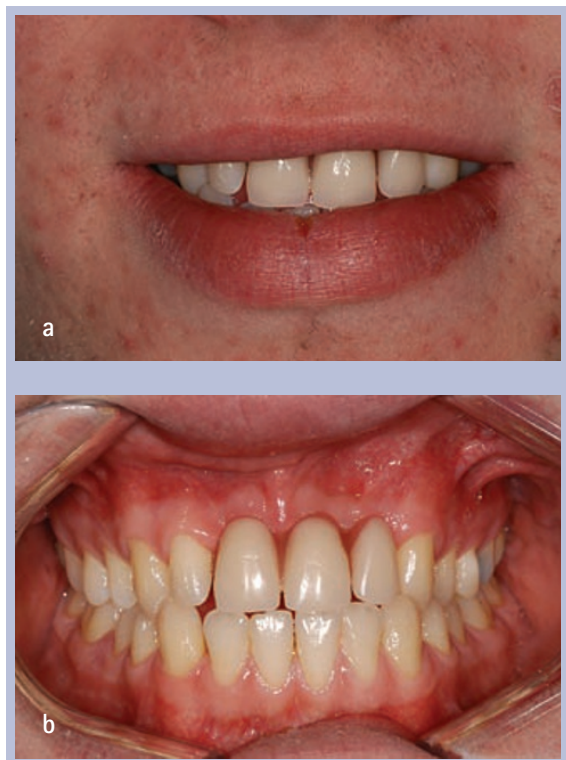

Fig. 4 A diagnostic denture. A: lips at rest. B: lips retracted showing gum fitted partial denture with ridge lap teeth producing an acceptable appearance

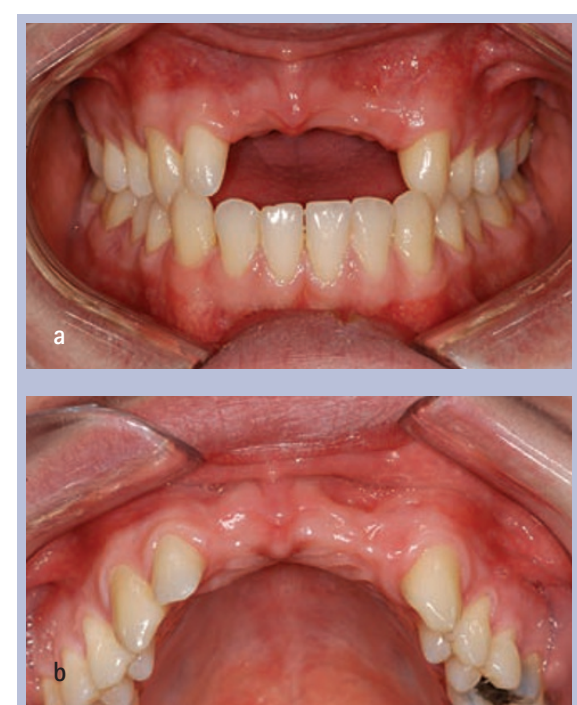

Fig. 5 The edentulous ridge in same patient as Figure 4. A: labial view showing slight loss of height in central part of ridge. Healthy keratinised gingiva is present. B: occlusal view suggesting adequate ridge width that may need to be verified using radiographic imaging 


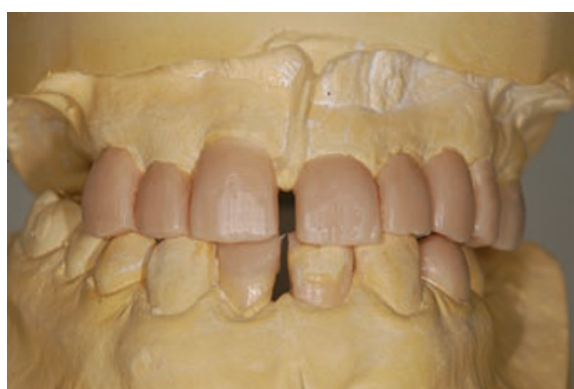

Fig. 6 A diagnostic wax-up on a cast

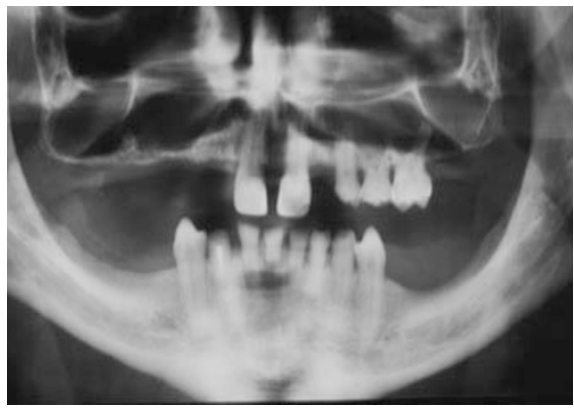

Fig. 7 The dental panoramic tomogram showing major anatomical structures, teeth and bone height in the edentulous zones. Measurements in the horizontal plane are not accurate and this radiograph provides no indication of ridge thickness

adequate number of implants within sound bone beneath the proposed location of the abutment teeth. In edentulous ridges bound by teeth, the available space will also be affected by angulation of adjacent tooth roots, which may be palpated and assessed radiographically. It is easy to damage adjacent roots during implant placement in tight spaces and this can precipitate early implant failure.

The occlusion should be carefully assessed, particularly in all excursive movements. It may be helpful to examine the occlusion with the existing prosthesis or the provisional prosthesis to assess the type of loading to which the implant restoration will be subjected.

\section{Study casts and diagnostic set-ups}

Study casts allow detailed measurements of many of the factors considered in the previous section. The proposed replacement teeth can be positioned on the casts by the technician using either denture teeth or teeth carved in wax (Fig. 6). The former have the advantage that they can be converted into a temporary restoration, which can be evaluated in the mouth by the clinician and patient. The diagnostic set-up therefore helps to determine the number and position of the teeth to be replaced and their occlusal relationship with the opposing dentition.

\section{Radiographic examination}

Radiographic examination is a central part of implant treatment from the planning phase to the long-term evaluation of treatment success. The most convenient overall radiographic examination is the dental panoramic tomogram (Fig. 7). This may need to be supplemented with intra-oral radiographs where the image quality does not permit proper assessment (Fig. 8). Periapical radiographs using a paralleling technique should be considered for all adjacent teeth, heavily restored teeth, teeth with known or suspected endodontic problems and teeth with moderate to advanced periodontitis.

Standard dental radiographs allow the clinician to make an initial assessment of the bone levels available for implant treatment, but as two-dimensional images they give no indication of bone width. In combination with clinical examination they may provide enough information to plan treatment without resorting to more complex imaging techniques. Tomographic examinations give cross-sectional and three-dimensional images. In addition to providing information about bone quantity they also provide some indication of the bone quality available, notably the thickness of the cortices as well as a measure of the density of the cancellous bone.

In order to facilitate planning using images at different magnifications, overlays depicting implants of various lengths and diameters at the corresponding magnifications can be superimposed directly on the radiograph (Fig. 8). These provide a simple method of assessing implant sites and implant placement at different angulations.

In order to optimise the information provided by more advanced radiographic techniques, it is helpful to provide information about the planned final restoration. A stent, which mimics the desired tooth set-up, is constructed and radiographic markers (eg gutta percha, amalgam) placed within it. Alternatively, if the patient has a suitable acrylic denture, radiographic markers may be placed within occlusal or palatal cavities cut in the acrylic teeth. The denture can also be replicated in acrylic with a radio-opaque medium to provide the radiographic stent.

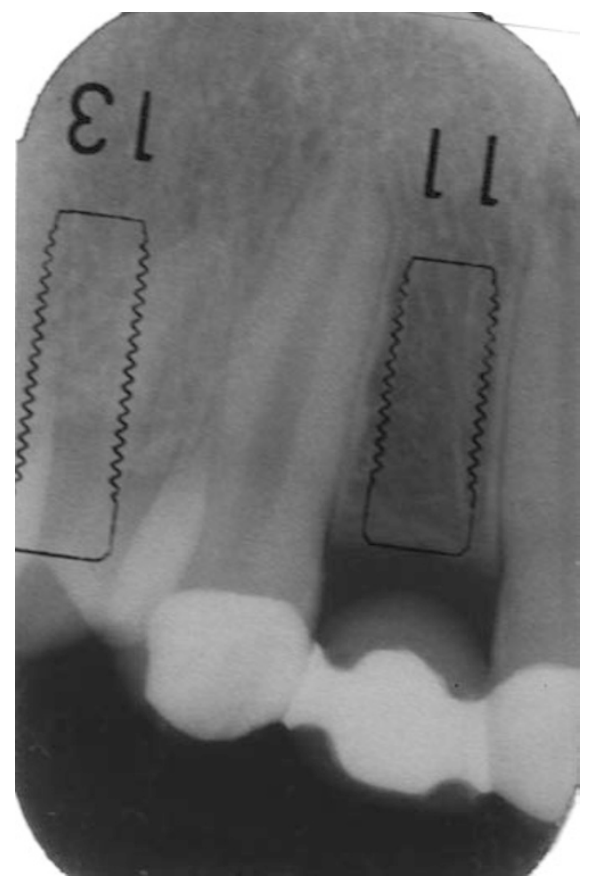

Fig. 8 An intra-oral radiograph of an upper lateral incisor space using a paralleling technique to check root alignment and available space. An implant overlay has been applied to judge what size of implant might be accommodated without damaging the adjacent teeth

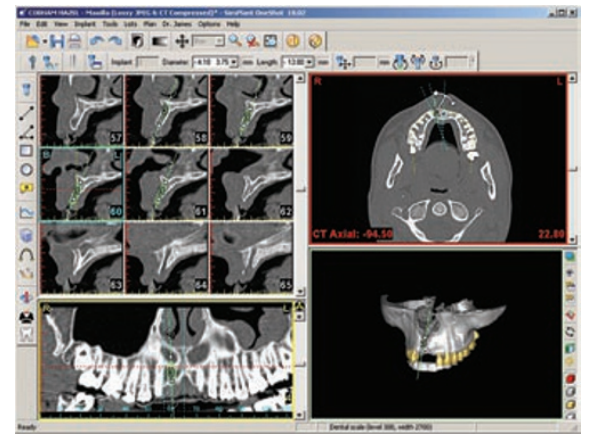

Fig. 9 An image from a CT scan using software to judge implant position within the bone in relation to a radiographic stent

CT scans are often used for complex cases, particularly for areas such as the posterior mandible to adequately locate the inferior dental canal and avoid damage to the neurovascular bundle during implant placement (Fig. 9). The quality of bone can also be assessed using CT scanning or clinically at the time of surgery. The most favourable quality of jaw bone for implant treatment is that which has a well formed cortex and densely trabeculated medullary spaces with a good blood supply. Bone which is predominantly cortical may offer good initial stability at implant placement but is more easily damaged by overheating during the drilling process, especially with sites more than 


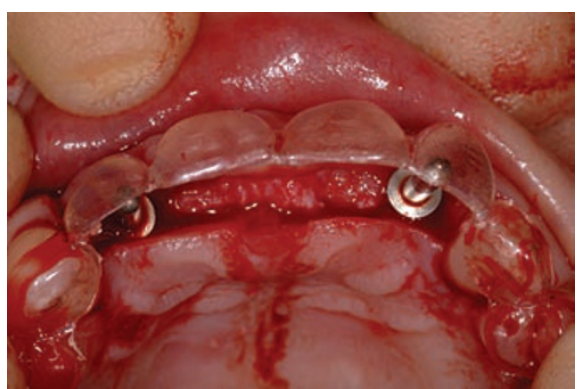

Fig. 10 A customised surgical stent used to help in implant positioning and angulation

\begin{tabular}{|c|c|}
\hline Prosthesis & $\begin{array}{l}\text { Suggested number } \\
\text { of implants } \\
\text { required }\end{array}$ \\
\hline \multicolumn{2}{|c|}{ Fixed restorations: anterior teeth } \\
\hline One missing tooth & 1 \\
\hline Two missing teeth & 2 \\
\hline Three missing teeth & 2 or 3 \\
\hline Four missing teeth & 2,3 or 4 \\
\hline \multicolumn{2}{|c|}{ Fixed restorations: molar teeth } \\
\hline One missing tooth & 1 or 2 \\
\hline Two missing teeth & 2 or 3 \\
\hline \multicolumn{2}{|l|}{ Full arch bridges } \\
\hline Edentulous maxilla & At least 6 \\
\hline Edentulous mandible & At least 4 \\
\hline \multicolumn{2}{|l|}{ Overdentures } \\
\hline Edentulous maxilla & At least 4 (joined) \\
\hline Edentulous mandible & 2 \\
\hline
\end{tabular}

$10 \mathrm{~mm}$ in depth. At the other extreme, bone with a thin or absent cortical layer and sparse trabeculation offers very poor initial implant stability and fewer cells with a good osteogenic potential to promote osseointegration.

Computer-based image software programs (eg Simplant) produce images of implants (and their restorative components) which can then be 'placed' within the CT scan. This enables the clinician to evaluate the relationships between the proposed implants and the ridge morphology, other anatomical features and adjacent teeth. When used in conjunction with a radiographic stent the possibility of reproducing the orientation envisaged at the planning stage is greatly increased. This has been developed to very high levels of sophistication using a combination of radio-opaque diagnostic set-ups, CT scans and stereolithic modelling. Using these techniques it is possible to provide:

- 3D models of the jaws

- Accurately fitting drill guides to assist the surgeon in placing the implant in the same position as that planned with the computer software

- Fabrication of fixed prostheses that can be fitted directly to the implants at the time of placement.

This rapidly developing area is of particular benefit in management of the more complex cases. It could make implant placement more precise and reduce the possibility of damage to important anatomical structures. It requires meticulous planning, a thorough understanding of the computer programme and available guides. At the clinical level there has to be sufficient intraoral space in a compliant patient to allow use of the technique and enough bone volume to allow for any errors

\section{IMPLANT PLACEMENT, NUMBERS, SIZE AND SPACING}

Implants should be placed using a careful aseptic surgical technique by a trained clinician with a trained assistant. Success is highly dependent upon a surgical technique which avoids heating the bone. Slow drilling speeds, the use of successive incrementally larger sharp drills and copious saline irrigation aim to keep the temperature below that at which bone tissue damage occurs (around $47^{\circ} \mathrm{C}$ for 1 minute).

The implants have to be placed at the correct positions, depths and angulations to allow fabrication of a functional and aesthetic prosthesis. Surgical stents/ guides can help considerably (Fig. 10). Poorly positioned or angled implants will compromise the prosthodontic reconstruction and in the worst cases the most highly skilled and ingenious of technicians will not be able to solve the problem. An adequate number of implants is required to support a given prosthesis (Table 2). The distribution of load to the supporting bone can be spread by increasing the number and dimensions (diameter, surface topography, length) of the implants. The spacing and three-dimensional arrangement of the individual implants will also be very important. The so-called 'tripod' arrangement of three implants is recommended in situations of high load, such

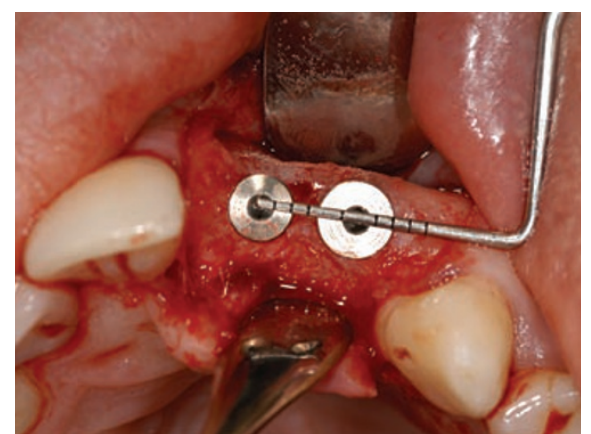

Fig. 11 Implants that have been placed close together. Space was available to place them further apart without being too close to the adjacent teeth

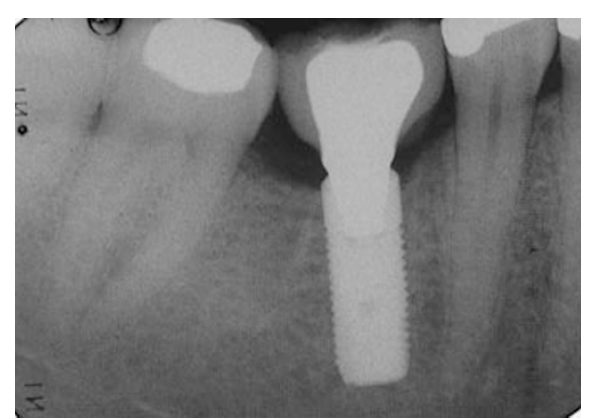

Fig. 12 A radiograph of a wide bodied $(5 \mathrm{~mm}$ diameter) implant used to replace a single molar tooth

as replacement of molar teeth in the partially dentate individual. Evidence for this is derived more from biomechanical theory than comparative clinical trials.

However, it is a great mistake to attempt to place too many implants in a given space (Fig. 11) and, if necessary, orthodontic treatment should be used to optimise spacing. The average implant is $4 \mathrm{~mm}$ in diameter, wide implants are $5 \mathrm{~mm}$ or greater in diameter and narrow implants less than $3.5 \mathrm{~mm}$. Wide diameter implants are of course stronger and provide a greater area for osseointegration. They are therefore particularly useful in molar replacement (Fig. 12). Narrow diameter implants are useful for narrow spaces and thin ridges but are weaker and may fail mechanically or biologically in high load situations. Some manufacturers publish warnings to this effect.

Spacing is required to provide:

- An adequate width of bone and soft tissue between implants and adjacent teeth

- For the prosthetic components not to impact on each other

- For the patient to be able to clean the prosthesis effectively. 
Implants placed next to natural teeth should allow an absolute minimum of $1 \mathrm{~mm}$ of intervening bone and preferably $2 \mathrm{~mm}$. It is advisable to allow a little more spacing between implant heads, ideally $3 \mathrm{~mm}$ and no less than $2 \mathrm{~mm}$. This is because in many systems the abutments are larger than the implant heads, and the restoration is often designed so that it increases in diameter to establish a good emergence profile. Connection of narrower abutments than the implant head allows for more soft tissue space and has been termed 'platform switching'. With all these factors competing for space it is easy to see how the soft tissue and oral hygiene may be compromised if implants are placed too close together.

The bone volume that can accommodate the proposed diameter and length of implant has to be determined radiographically. Implants should be selected to ensure good initial stability, but in some instances the clinician is limited by the need to avoid damage to important anatomical structures, such as the inferior dental nerve. The assessment of length should allow an adequate safety margin, particularly as most drills are designed to prepare the implant site slightly longer than the chosen implant.

\section{TREATMENT PLANNING}

It is imperative to consider all treatment options with the patient, and during detailed planning it may become apparent that an alternative solution is preferred. In all cases the implant treatment should be part of an overall plan to ensure the health of any remaining teeth. Once the goal or end point has been established it should be possible to work back to formulate the treatment sequence. The cost of the proposed treatment plan is also of great relevance, and this may therefore place limits on treatment options. The advantages and disadvantages of the various options can be presented to the patient following careful clinical and radiographic examinations as follows.

\section{Removable prostheses}

These are a commonly prescribed treatment option and may be used as a longterm restoration or provisional restoration before a fixed prosthesis.

Advantages:

- Replace multiple teeth in multiple sites
- Support obtained from mucosa and/ or teeth

- Generally do not require extensive preparation of abutment teeth

- May be designed to accommodate future tooth loss

- Can be used to replace missing soft tissue

- Can provide good lip support by incorporating labial flanges

- Aesthetics may be very good

- The least expensive of restorations.

Disadvantages:

- Removable prostheses may not be liked by the patient and may reduce self-confidence

- Connectors cover soft tissue such as the palate and gingiva

- In subjects with less than ideal oral hygiene they may compromise the health of the periodontal tissues and promote caries around abutment teeth

- Retentive elements such as clasps may spoil aesthetics

- Moderate maintenance requirements and durability.

\section{Fixed prostheses}

Fixed prostheses fall into two main categories:

1. Resin bonded bridgework

2. Conventional partial or full coverage bridgework.

\section{Resin bonded bridgework}

\section{Advantages:}

- Minimal or no preparation required

- Fixed restoration

- Good aesthetics if ideal spacing exists and abutment teeth are satisfactory

- Less expensive than conventional bridges

- Consequence of failure are relatively small - caries is readily diagnosed in most instances. Cantilever designs for single tooth replacements minimise potential problems.

Disadvantages:

- Lack of predictability: decementation leading to loss of retention or caries under one of the retainers - average life span 5-7 years

- Dependent upon meticulous technique and available enamel/surface area for bonding
- Change in colour/translucency of abutment teeth due to presence of retainer

- May interfere with occlusion, particularly incisal guidance

- Patients may feel sense of insecurity with restoration, especially if their bridge has debonded previously.

Conventional partial or full coverage bridgework

Advantages:

- Fixed

- Good appearance, including that of abutment teeth if they need to be improved/harmonised

- Medium term predictability is good for short span bridges

- Good control of occlusion possible

- Minimally compromise oral hygiene.

Disadvantages:

- Involve considerable tooth preparation which sometimes results in pulpal sequelae

- Failure due to decementation and caries of abutment teeth may lead to further tooth loss

- Moderately expensive

- Highly operator-dependent requiring exacting techniques both clinically and technically

- Requires lengthy clinical time and temporary restorations

- Irreversible.

\section{Implant retained prostheses}

Advantages:

- Fixed or removable

- Independent of natural teeth - can provide fixed restoration where no abutment teeth exist

- Immune to dental caries

- High level of predictability

- Good maintenance of supporting bone.

Disadvantages:

- Dependent upon presence of adequate bone quantity and quality

- Involves surgical procedure(s)

- Highly operator/technique-dependent

- High initial expense and lengthy treatment time

- Moderate maintenance requirements especially for removable or extensive fixed prostheses. 


\section{Treatment choices}

In situations where all types of prosthesis are possible, the final choice may rest with the patient and is largely dependent upon their expectations/desires, financial budget and willingness to undergo treatment. It is important that the patient's expectations are realistic and achievable. However, some factors may dictate that a certain type of restoration is not feasible or is undesirable.

The treatment plans should be outlined in writing and an estimate of the relative costs given. Complex treatment plans require more detailed descriptions and a projected timetable for completion and costings. It is important to ensure that the patient understands the proposals and is given the opportunity to clarify any matters. A written consent to the agreed treatment plan is essential.

\section{SUCCESS/SURVIVAL RATES}

There are suggested criteria for what constitutes a successful implant. Some criteria are focused on clinical/radiographic factors such as immobility, absence of radiolucencies and good maintenance of bone levels. Patient-based criteria such as absence of pain and nerve damage and provision of a functional and aesthetic prosthesis must be achieved to satisfy most patients.

Some patients may want to know the life expectancy of the dental implant treatment that is proposed. Although there are many published studies, they are normally from specialised centres or by expert practitioners. Many studies also report similarly high figures when more risky protocols such as immediate extraction and implant insertion or immediate placement and loading are used. It is important to appreciate that the criteria for adopting these protocols are often very stringent, with many patients not meeting the inclusion criteria set by very experienced clinicians. You should be realistic when quoting success/survival figures to patients and ideally base this on your own experiences or audit. It is crucial to recognise factors that may increase failure (Table 3) and to develop skills and high success rates on straightforward standard protocols before venturing into the more demanding ones.

The original osseointegrated implants such as the Branemark system showed that implants placed in the
Table 3 Local factors that may increase implant failure

Contamination of implant at placement o

subsequent infection

Poor initial stability:

Due to technique used

Due to inadequate implant

dimensions

Due to poor bone quality

Placement in previously irradiated bone

Placement in an area of infection

Placement in previously grafted bone

Placement with simultaneous bone grafting

Subjected to early/poorly controlled loading

Overheating of the bone during surgical prepara tion of the site

Injury to adjacent periodontal ligament of a neighbouring tooth

mandible (particularly anterior to the mental foramina) enjoyed a higher success rate than the maxilla (approximately 95\% success for implants in the mandible compared with $85-90 \%$ for the maxilla). An example of the lowest recorded success rates were for short implants $(7 \mathrm{~mm})$ used in the maxilla to support overdentures, especially when the implants were not joined together. Further studies showed that the overall mean failure rate in smokers was about twice that in non-smokers. Development of implant systems over the last decade, particularly with refinement of implant surfaces (see below), have led to significantly lower rates of early failure and better long-term success. Some studies have suggested that these improvements apply equally to smokers, with near equivalent success rates to non-smokers. However, it is recommended that smoking should still be considered a significant risk factor and patients warned of this association. The overall lifetime exposure of the patient to smoking (eg pack years number of packs per day $\times$ number of years smoking) is important with regard to the chronic detrimental effects on the healing potential and inflammatory and immune responses. It should also be noted that reported failure rates are not evenly distributed throughout the patient population. Rather, implant failures are more likely to cluster in certain individuals.

The success rates for prostheses at 5 and 10 years are somewhat lower (approximately $85 \%$ ). This is due to occasional failure of implants but more commonly technical complications such as chipping of the porcelain, loss of retention, screw loosening or screw fracture. Patients need to be made aware that all prostheses will require some level of maintenance and on some occasions replacement of the prosthesis will be required.

Carefully planned functional occlusal loading will result in maintenance of osseointegration and possibly increased bone to implant contact. In contrast, excessive loading may lead to bone loss and/or component failure. Clinical loading conditions can vary from a single tooth replacement in the partially dentate case to a full arch reconstruction in the edentulous individual. Implants which support overdentures may present particular problems with control of loading as they may be largely mucosal supported, entirely implant supported or a combination of the two. The lack of mobility in implant supported fixed prostheses requires provision of shallow cuspal inclines and careful distribution of loads in lateral excursions. With single tooth implant restorations it is important to develop initial tooth contacts on the natural dentition and to carefully control guidance in lateral excursions on the implant restoration. Loading will also depend upon the opposing dentition which could be natural teeth, another implant supported prosthesis or a conventional removable prosthesis. Surprisingly high forces can be generated through removable prostheses. Great caution should be exercised in treating patients with known parafunctional activities such as bruxism. They are more likely to produce loads that can result in biological and or biomechanical failure of the implants, components and prosthetic reconstructions.

\section{RE-EVALUATION OF THE IMPLANT RETAINED PROSTHESIS}

It is generally recommended that patients treated with implant prostheses are seen at least on an annual basis, but in many cases they will also require routine hygienist treatment at 3, 4 or 6 monthly intervals according to individual requirements.

\section{Clinical evaluation}

The presence of inflammation of the soft tissue (peri-mucositis) or inflammation 
with loss of bone (peri-implantitis) should be noted and treated. Probing depths (and/ or clinical attachment loss) and bleeding on probing can be recorded. Probing depths $5 \mathrm{~mm}$ or greater with bleeding or exudate are more likely to be associated with peri-implantitis. If this is not treated it could lead to progressive bone resorption and loss of the implant.

\section{Radiographic evaluation}

Baseline radiographs to show crestal bone levels and the state of the peri-implant bone should be taken as part of normal documentation at the time of fitting the final prosthesis. These should be repeated on an annual basis for the first 2 or 3 years to establish that the bone levels are stable. It should be remembered that some initial bone loss may occur during the first year of function with some implants, but that a steady state should then be established thereafter. The interval between radiographs may be extended if the bone appears stable over the first few years of function

Radiographs play an important part in the successful planning and execution of implant treatment. It is important to have an understanding of the different techniques available and their appropriate application. They are an important part of the patient's records and as such constitute a significant proportion of the medico-legal documentation of the patient. It is the responsibility of the clinician to ensure that radiographs are appropriate, readable and are retained and repeated at accepted intervals throughout treatment and follow-up.

\section{TRAINING AND GDC GUIDELINES FOR PRACTITIONERS PROVIDING IMPLANT TREATMENT}

The General Dental Council (www.gdc-uk. org) in conjunction with the Faculty of General Dental Practitioners (UK) have published guidelines on training in implant dentistry for general dental practitioners (www.fgdp.org.uk). This was in response to growing concern about the delivery of implant treatment in general dental practice. Basically, 'The GDC and the working group wanted to ensure patient protection by establishing and maintaining standards of training in implant dentistry.' There were a number of important fundamental recommendations:

'Before starting to place implants a general dental practitioner should have practised clinical assessment, treatment planning, and the placement of implants in the presence of an experienced implant clinician, as part of a course in implant dentistry meeting these standards. This mentoring should be continued until the experienced implant clinician considers the practitioner to be competent.'

In addition the guidelines make specific recommendations about more advanced treatment modalities involving bone grafts:

'Before progressing onto this type of advanced surgery a person must be competent and experienced in the placement of implants as described above. The placement of implants with bone augmentation or minor modification of anatomical structures demands a high level of surgical experience. The ability of a person to do such treatment should have been mentored and formally assessed by a suitably competent and experienced individual. The person must have attended courses which specifically train in these techniques and include an element of formal assessment. The person must be competent to deal with immediate and long-term complications of the treatment provided:

It is proposed that these guidelines will be reviewed and updated in line with developments in implant dentistry education and clinical activity within the UK.

\section{Mentoring}

Guidance on implant training is unique in advising that clinicians who wish to place implants surgically ought to have been mentored. Mentoring tends to be longerterm, more informal, and with a wider focus. ${ }^{1}$ Mentoring is flexible and driven by the needs of the mentee for guidance and support at any moment in time. In this relationship the mentor passes on knowledge and experience, and generally relies upon having had similar experiences and hence an empathy with the mentee and a close personal understanding of the relevant, specific training and development issues involved. This is not necessarily so in the case of coaching. Both mentoring and coaching allow the assisted individual to develop their skills and reach their full potential by encouraging, supporting and inspiring them and drawing out the skills of this junior party.

Each individual practitioner has a duty of care to each and every patient in whose treatment they are involved. This duty of care has an ethical as well as a legal dimension and reflecting this fact, one should start from the premise that such a duty of care exists, even when one is not treating the patient personally. Thus, while a mentor's relationship with the colleague they are mentoring will be self-evident, one should not lose sight of the more subtle and indirect relationship with any patients involved under the care of the mentee, and in relation to whom the mentor might be providing advice.

Depending upon the extent to which there can be shown to be a "master and servant' relationship between the mentor and mentee, it may be possible to argue that the mentor is in some respects vicariously viable for the negligent acts and omissions of the mentee. In a 'true' mentoring arrangement, however, it should be clear (and separately confirmed in any relevant documentation regarding the arrangement) that there is no such relationship between the parties because the mentee is autonomous, working independently and electing to call upon the advice and guidance of the mentor only as and when he/she chooses to, reflecting their own assessment of the need for such assistance. In such circumstances it would be extremely difficult to demonstrate vicarious liability on the part of the mentor.

However, a mentor should not assume from this that they could never be held to be liable for any adverse outcome as a result of the mentee's treatment of a patient. In most cases it is fair to say that they would not, but there will be circumstances in which a mentor could be drawn into civil proceedings such as a negligence claim, or into a GDC complaint/investigation. In the former, the mentor could be named as a primary defendant or codefendant, or alternatively, could be the subject of a 'third party' action, perhaps brought by a mentee who feels aggrieved that s/he has followed the mentor's advice and guidance and has ended up being sued as a result of this.

\section{CONCLUSIONS}

- Obtain adequate training and work 
within your level of experience and skill

- Evaluate and treatment plan carefully

- Communicate with the patient and dental team, especially the highly skilled technician

- Document carefully and retain all relevant radiographs and casts

- Adopt protocols that are proven and predictable - do not cut corners

- Provide a high level of care and skill

- Refer patients that are too complex.
Thanks to the editor of the series Len D'Cruz who contributed to the section on Mentoring. Some of this material has previously appeared in 'A Clinical Guide to Implants in Dentistry' BDJ Books 2008.

1. Dental Protection Ltd. Position statement: mentoring. London: Dental Protection Ltd, 2008. http:// www.dentalprotection.org/uk/news/positionstatements/mentoring (accessed 30 March 2010). 